\title{
DETERMINAÇÃO VIA CFD DE CAMPOS DE TEMPERATURA EM CÂMARAS DE ARMAZENAMENTO
}

\author{
G. P. DAMASCENO, J. L. G. CORRÊA e F. R. NASCIMENTO \\ ${ }^{1}$ Universidade Federal de Lavras, Departamento de Ciência dos Alimentos \\ E-mail para contato: gutrunks@yahoo.com.br
}

\begin{abstract}
RESUMO - A manutenção da qualidade e da vida de prateleira podem ser obtidos em câmaras de armazenamento refrigerado. $\mathrm{O}$ objetivo deste trabalho foi o estudo de dois casos de armazenamento de uvas em câmara com o auxílio da fluidodinâmica computacional (CFD). Os dados preditos foram validados por dados experimentais coletados em dezesseis pontos diferentes da câmara. Nos dois casos, observaram-se a formação de diferentes regiões com relação ao fluxo de ar e temperatura.
\end{abstract}

\section{INTRODUÇÃO}

O emprego da fluidodinâmica computacional, CFD, pode auxiliar na compreensão do desempenho da refrigeração das câmaras de armazenagem e da distribuição do fluxo de ar e temperatura dentro das mesmas (Chourasia e Goswami, 2007, Delele et al., 2013). Xie et al. (2006) mostraram que um escoamento ideal garante a distribuição uniforme do ar frio, reduz a perda de peso do produto, melhora a transferência de calor entre o meio, economiza energia e mantém a qualidade do produto, enquanto estocado.

O objetivo deste trabalho foi o estudo de caso de armazenamento de uvas em câmara com o auxílio da fluidodinâmica computacional (CFD) com a identificação dos campos de temperatura e fluxo de ar. Testou-se a câmara com 90 e $100 \%$ de sua capacidade total de armazenamento com a validação por dados experimentais do $2^{\circ}$ caso.

\section{MATERIAL E MÉTODOS}

Os dados experimentais foram obtidos em uma instalação localizada na região do Vale do São Francisco (Petrolina, PE). Uvas frescas foram embaladas em caixas e empilhadas em paletes. A temperatura média de bulbo seco externo, $35^{\circ} \mathrm{C}$ e de bulbo úmido $25^{\circ} \mathrm{C}$. Os cálculos para propriedades e carga térmica foram segundo os padrões estabelecidos pela "American Society of Heating, Refrigerating and Air Conditioning Engineers" (ASHRAE, 2010).

As medidas externas da câmara são $12,7 \mathrm{~m}$ x 13,75 m x 3,5 m ( $\mathrm{L} \times \mathrm{C} \times \mathrm{A})$ com paredes revestidas (100 $\mathrm{mm}$ de poliuretano expandido), capacidade total de armazenamento, 100 paletes i.e., $85.000 \mathrm{~kg}$ de uva. A temperatura de armazenamento foi $0{ }^{\circ} \mathrm{C}$. As dimensões de paletização foram $1,0 \mathrm{~m}$ x 1,2 m x 2,4 m ( $\mathrm{L} \times \mathrm{C} \times \mathrm{H})$. Os dados experimentais foram obtidos com auxílio de um anemômetro (VT 100, 0,15 a 3,00 m.s ${ }^{-1}$ desvio $0,05 \mathrm{~m} \cdot \mathrm{s}^{-1},-20,0$ a $80,0{ }^{\circ} \mathrm{C}$, desvio $0,3^{\circ} \mathrm{C}$ ).

Na configuração da câmara totalmente carregada com os pontos dos dados experimentais estão 
representados conforme a Figura 1 vista A. A visão frontal B da câmara apresenta a altura de obtenção dos dados, sendo que os pontos 1, 3, 7, 10, 14 e 16 ficaram $280 \mathrm{~cm}$ acima do solo, portanto acima dos paletes, e o restante a $160 \mathrm{~cm}$ do solo. Nos pontos acima dos paletes, considerando a direção y, suas leituras foram feitas aproximadamente $25 \mathrm{~cm}$ de distância da parede externa. A leitura para os outros pontos foi realizada segundo suas posições na Figura 1 vista A, pontos 2, 5, 12 e 15 próximos ao centro do corredor e pontos 4, 6, 8, 9, 11 e 13 entre os paletes que foram delimitados pelos círculos. Dentro da câmara fria, os paletes ficaram dispostos em colunas na direção x, a $15 \mathrm{~cm}$ de distância das paredes externas e 5 e $30 \mathrm{~cm}$ entre eles; ficaram dispostos em linhas na direção y, a $14,5 \mathrm{~cm}$ de distância das paredes externas e 10 e $161 \mathrm{~cm}$ entre eles; na direção z a distância dos paletes em relação ao solo foi de $10 \mathrm{~cm}$. Estes espaçamentos visaram uma melhor distribuição do fluxo de ar e troca de calor mais eficiente entre produto e meio. Foi estabelecido para o corredor central um espaçamento mínimo de $160 \mathrm{~cm}$ na direção y para o deslocamento seguro de uma empilhadeira dentro da câmara. Na configuração com $90 \%$ da carga, as duas linhas de paletes localizadas abaixo dos evaporadores foram retiradas.

O somatório final do cálculo de carga térmica total de refrigeração foi de $501 \mathrm{~kW}$. A partir deste dado foi escolhido um evaporador que fornece uma vazão mássica de $4,20 \mathrm{~kg} \cdot \mathrm{s}^{-1}$ de ar distribuídos igualmente por três sopradores, sendo necessário dois evaporadores para atender a demanda de energia.

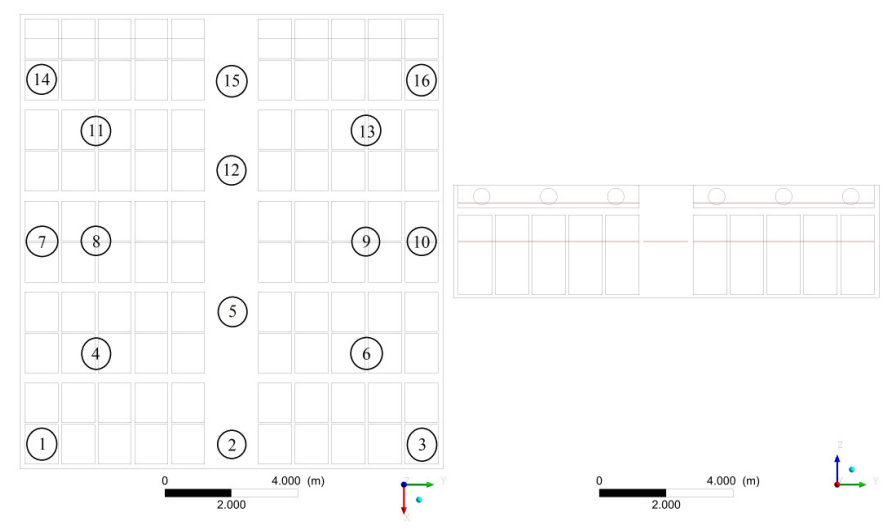

Figura 1 - Localização de dados experimentais na câmara fria. Vista superior A dos pontos de leitura e vista frontal B da altura de leitura.

As geometrias foram confeccionadas com auxílio do código comercial ICEM ${ }^{\circledR}$ 11.0, com malhas hexaédricas. Foram realizados testes de independência de malhas utilizando as variáveis velocidade e pressão média na saída e entrada dos evaporadores para garantir que os resultados obtidos seriam independentes da resolução das malhas. A malha para simulação no caso com $90 \%$ de carga apresentou 687.739 células e no caso com $100 \%$ de carga, 682.056 células ou elementos.

Foram estabelecidas as condições de contorno para as duas geometrias: paredes, entradas e saídas. Para o primeiro e segundo casos considerou-se respectivamente que nas paredes ocorreu uma transferência de calor entre o meio externo e interno de $14,8 \mathrm{~W} \cdot \mathrm{m}^{-2}$ e nos paletes, do produto para o meio de $8,9 \mathrm{~W} \cdot \mathrm{m}^{-2}$ e $9,9 \mathrm{~W} \cdot \mathrm{m}^{-2}$, sendo que para a influência da parede no fluido se aplicou a condição de não deslizamento. Nas condições de entrada foi estabelecido para cada um dos seis sopradores a taxa de vazão mássica de ar de $1,4 \mathrm{~kg} . \mathrm{s}^{-1}$ e para as condições de saída em cada um dos evaporadores a taxa mássica de 4,2 kg. $\mathrm{s}^{-1}$. As condições iniciais foram temperatura de armazenamento constante e igual a 0 
${ }^{\circ} \mathrm{C}$ e fluido contínuo ar a $0{ }^{\circ} \mathrm{C}$. Considerou-se regime de escoamento transiente, passo de tempo de $5 \times 10^{-3}$ a $1 \times 10^{-2} \mathrm{~s}$ e critério de convergência por resíduo do tipo RMS com valor de $1 \times 10^{-4}$.

A região de domínio do escoamento do fluido foi delimitada pela geometria e empregou-se nas simulações o modelo de turbulência SST desenvolvido por Menter (1993) e de total energia para transferência de calor, selecionados de trabalho realizado anteriormente (Damasceno et al., 2013).

\section{RESULTADOS E DISCUSSÃO}

Nas simulações dos dois casos os resíduos médios (RMS) atingiram valores abaixo do critério de convergência estabelecido para as equações de transporte, energia e turbulência, ou seja, abaixo de $1 \times 10^{-4} \mathrm{e}$ os desequilíbrios no domínio para as condições de contorno estabelecidas ficaram abaixo de $1 \%$, o que implica que o primeiro passo para se obter resultados satisfatórios através das simulações foi alcançado com sucesso.

De acordo com um trabalho anterior (Damasceno et al., 2013), os dados preditos para o segundo caso proposto neste estudo, no qual a câmara de armazenamento está totalmente carregada, apresentaram erro geral médio de $27,1 \%$ para velocidade de escoamento do ar e de $16,2 \%$ para a temperatura em relação aos dados experimentais dos pontos de estudo. Considera-se que a predição foi bastante satisfatória para simulação em escala industrial pois trabalhos realizados em escalas piloto (Delele et al., 2009, Delele et al., 2013) apresentaram erro médio de predição de 18,5\% para a velocidade do ar e de $17,13 \%$ para a temperatura.

Obtiveram-se dados experimentais com a carga total da câmara e optou-se por simular a câmara com $90 \%$ de sua capacidade para a verificação dos campos de velocidade e temperatura nesta condição. Os sopradores presentes em cada evaporador possuem o papel de injetar constantemente o ar frio dentro da câmara e os paletes localizados próximos e logo abaixo da linha dos sopradores segundo Ho et al. (2010) auxiliam na obtenção de maior uniformidade de temperatura e no resfriamento. Na figura 2 pode-se notar a diferença nas linhas de escoamento do ar para cada configuração.
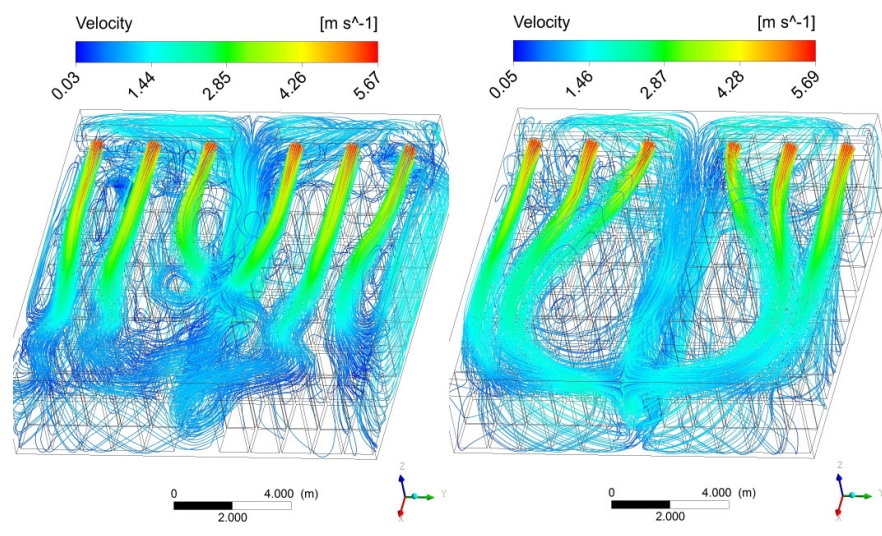

Figura 2 - Linhas de escoamento do ar no caso 1 (esquerda) e no caso 2 (direita).

Para o mesmo modelo e condições adotadas nos dois casos, as linhas de escoamento apresentaram variação na velocidade local de escoamento menor que $0,3 \%$, nos dois casos ocorreu predominância do escoamento para o corredor central, porém no caso 1 as linhas se espalharam de 
forma mais uniforme na parte superior e bordas laterais na direção x, mas com menor velocidade ao final deste percurso superior se comparadas às linhas do caso 2. A única diferença existente nas geometrias estava na ausência da linha de paletes abaixo dos evaporadores para o caso 1, sendo que a desigualdade no número de células ou elementos de cada configuração se deve a isto, pois os refinamentos foram feitos de maneira semelhante para padronizar esta etapa do processo. Uma explicação para estes resultados preditos pode estar na própria teoria aplicada aos efeitos turbulentos aparentes nas simulações, de acordo com o software ANSYS CFX (2007) as equações deste modelo representam as quantidades de fluxo médio apenas, enquanto se modela os efeitos de turbulência, ou seja, foram empregados procedimentos de média estatística para obtenção destas equações, o que pode implicar nas diferenças para cada caso simulado.

Na sequência foram analisados os campos de velocidade e temperatura obtidos através dos planos $\mathrm{xy}, \mathrm{zx}$ e yz, delimitando áreas que englobassem todos os pontos preditos. No plano xy as áreas de interesse abrangeram todos os dados preditos, conforme as figuras 3 e 4 os casos 1 e 2 podem ser observados separadamente a 1,60 e 2,80 m.
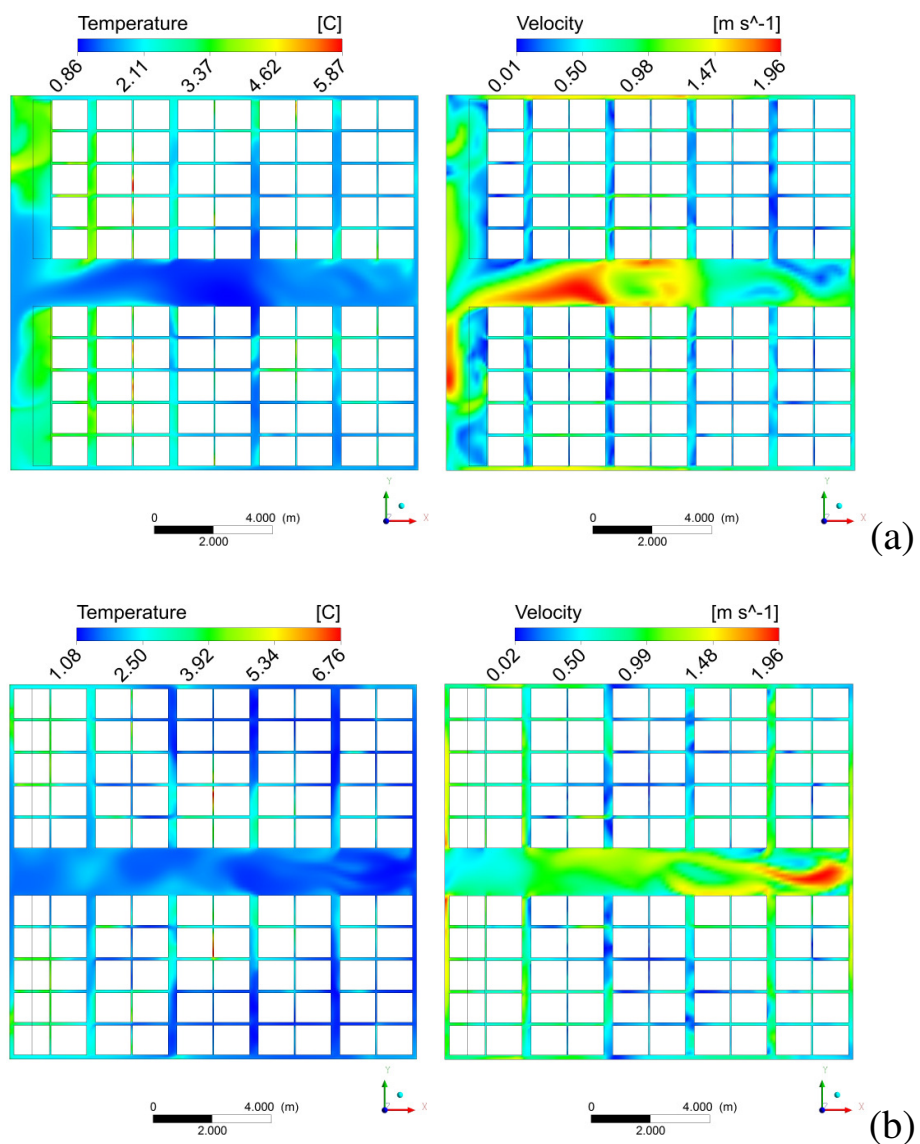

Figura 3 - (a) Campos de velocidade e temperatura do caso 1 a 1,60 m de altura e (b) Campos de velocidade e temperatura do caso 2 a 1,60 $\mathrm{m}$ de altura. 


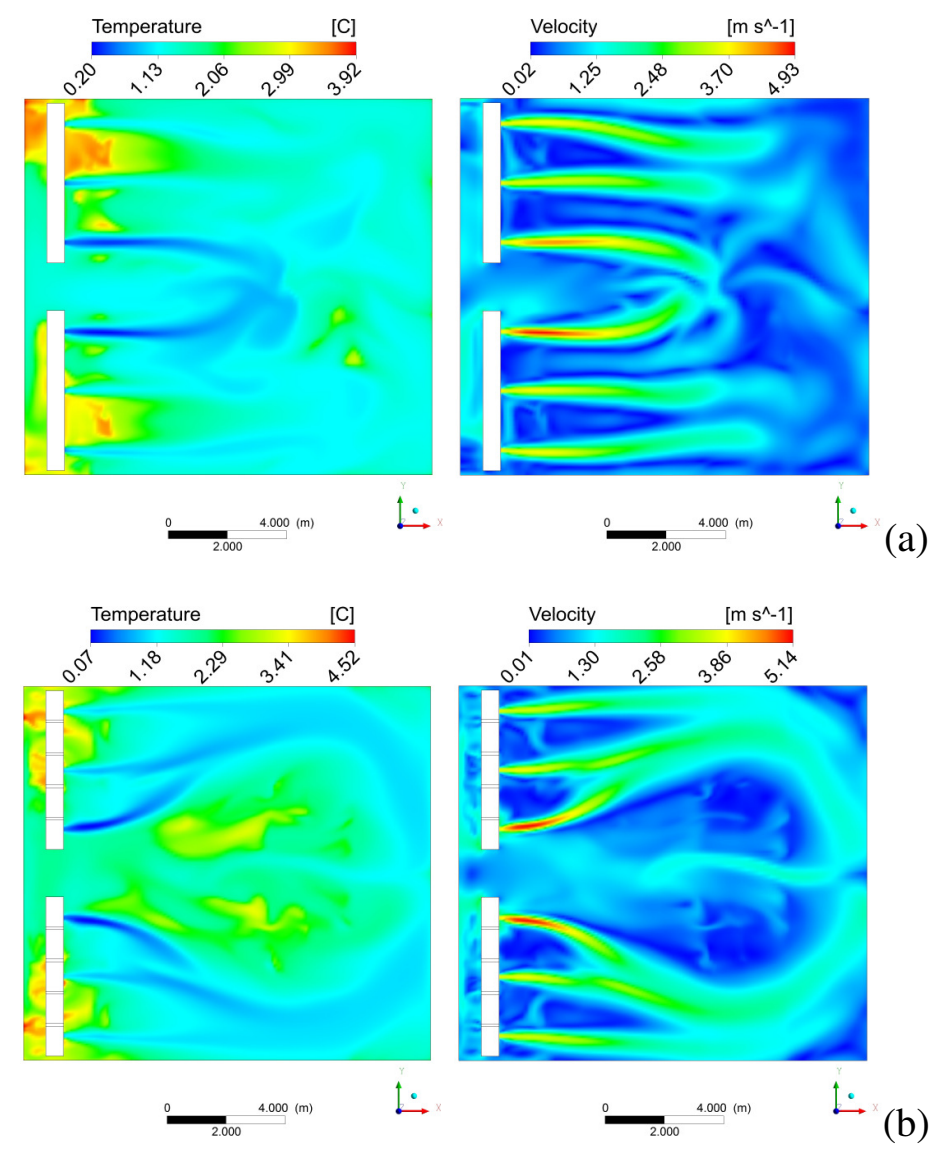

Figura 4 - (a) Campos de velocidade e temperatura do caso 1 a 2,80 $\mathrm{m}$ de altura e (b) Campos de velocidade e temperatura do caso 2 a 2,80 $\mathrm{m}$ de altura.

Uma diferença aparente ocorreu na velocidade de escoamento no corredor central, sendo que para o caso 1 (figura 3a) as maiores velocidades prevaleceram no início do corredor e para o caso 2 (figura 3b) ao final, e estas desigualdades se refletiram nos dados preditos com variações de 0,40 a $0,82 \mathrm{~m} \cdot \mathrm{s}^{-1} \mathrm{e}$ máxima de $0,5^{\circ} \mathrm{C}$. Também a distribuição dos paletes ao longo da câmara torna-se um parâmetro de análise substancial, pois no menor espaçamento, de $5 \mathrm{~cm}$ na direção $\mathrm{x}$, ocorreram as maiores variações de temperatura, no estudo em escala piloto de Tanaka et al. (2010) que buscou melhorar a performance de refrigeração de uma câmara parcialmente carregada estes obtiveram os melhores resultados para cargas empilhadas com menores alturas e espaçadas $15 \mathrm{~cm}$ entre si.

Para os dois casos pode se observar que nas regiões com maior velocidade de escoamento predominaram menores temperaturas. Os dados preditos para os pontos localizados no centro e final das câmaras (figura 4) apresentaram variações na velocidade e temperatura de $0,01 \mathrm{a} 0,28 \mathrm{~m} \cdot \mathrm{s}^{-1} \mathrm{e}$ máxima de $0,2^{\circ} \mathrm{C}$, com exceção da velocidade no ponto 7 que variou $0,82 \mathrm{~m} \cdot \mathrm{s}^{-1}$; os pontos próximos aos sopradores apresentaram baixa velocidade de escoamento e variação média de $0,7^{\circ} \mathrm{C}$.

No plano zx as áreas entre os paletes, conforme as figuras 5 e 6 , apresentam os casos 1 e 2 separadamente na direção y a 2,25 m abrangendo os pontos 4,8 e 11 e 10,45 m abrangendo os pontos 6,9 e 13. 


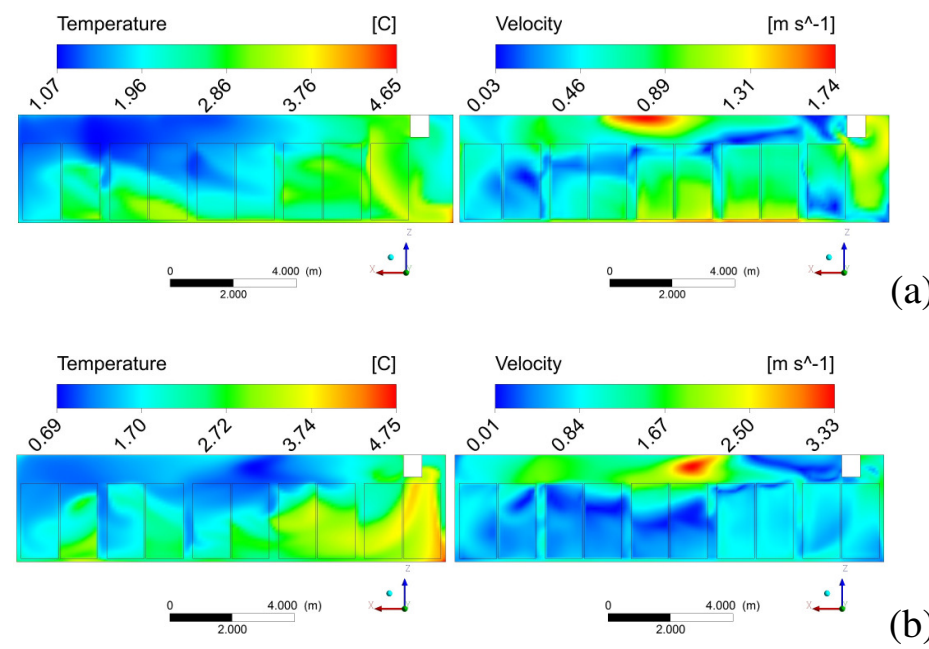

Figura 5 - (a) Campos de velocidade e temperatura do caso 1 a 2,25 $\mathrm{m}$ da origem em y e (b) Campos de velocidade e temperatura do caso 2 a 2,25 $\mathrm{m}$ da origem em $\mathrm{y}$.
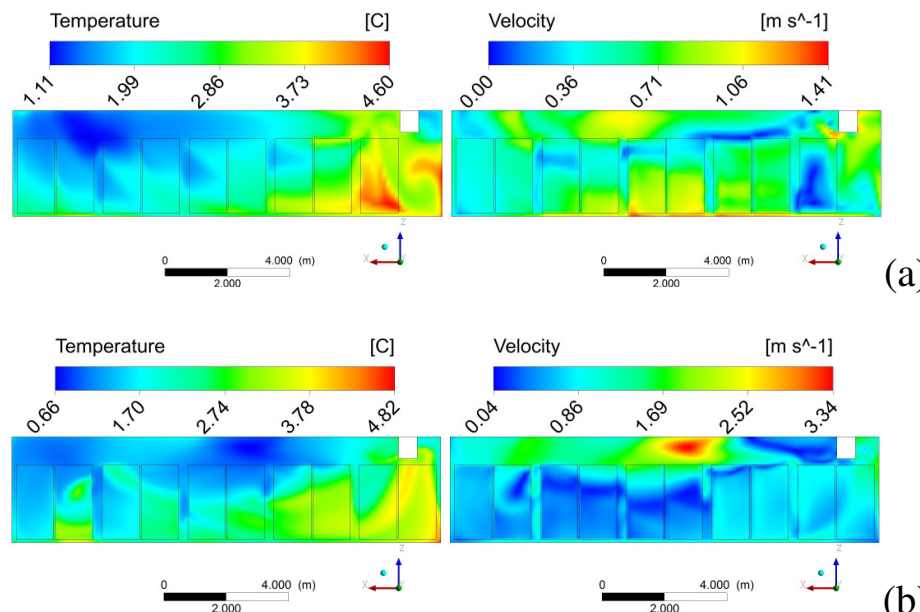

(a)

Figura 6 - (a) Campos de velocidade e temperatura do caso 1 a 10,45 $\mathrm{m}$ da origem em y e (b) Campos de velocidade e temperatura do caso 2 a 10,45 m da origem em y.

No geral a distribuição do fluxo de ar e a variação de temperatura apresentaram um padrão comum para os dois casos em ambos os lados (figuras 5 e 6), pois a baixa velocidade que se iniciou nos sopradores e seguiu até a parte inferior no final da câmara delimitou duas regiões: uma superior com menores temperaturas e uma inferior com maiores temperaturas, diferenciando-se em focos mais isolados. Este padrão se seguiu nos pontos e as temperaturas aumentaram do fundo para a entrada abaixo dos evaporadores, com exceção do ponto 9 no caso 2 , se comparado ao caso 1 a temperatura variou próximo de $0,9^{\circ} \mathrm{C}$.

No plano yz as áreas entre os paletes, conforme as figuras 7 e 8 , apresentam os casos 1 e 2 separadamente na direção x a 5,65 m e 6,86 m abrangendo os pontos 7, 8, 9 e 10 . 


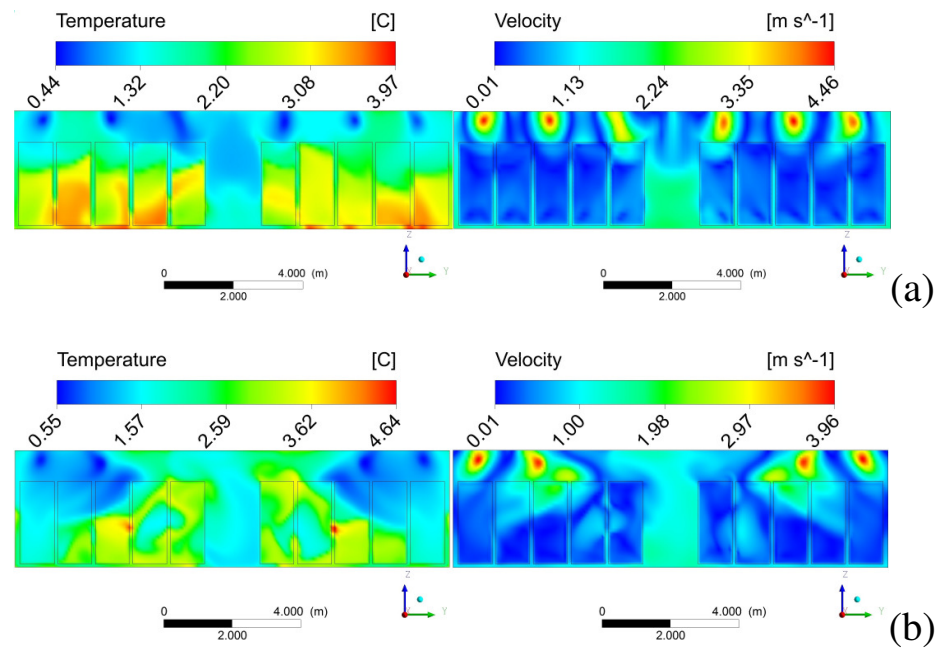

Figura 7 - (a) Campos de velocidade e temperatura do caso 1 a 5,65 $\mathrm{m}$ da origem em $\mathrm{x}$ e (b) Campos de velocidade e temperatura do caso 2 a 5,65 $\mathrm{m}$ da origem em $\mathrm{x}$.

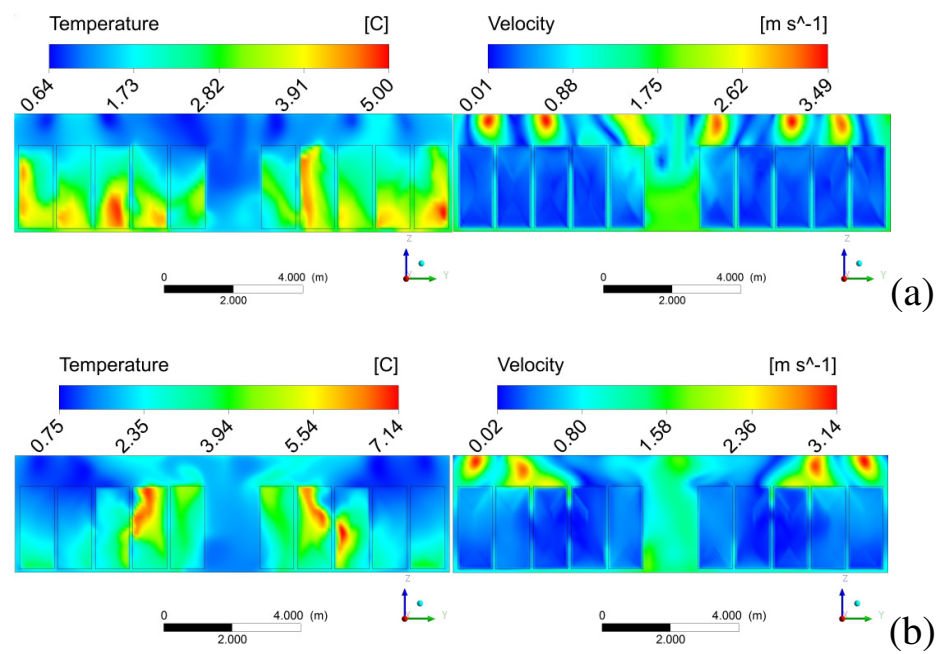

Figura 8 - (a) Campos de velocidade e temperatura do caso 1 a 6,86 m da origem em $\mathrm{x}$ e (b) Campos de velocidade e temperatura do caso 2 a $6,86 \mathrm{~m}$ da origem em $\mathrm{x}$.

A maior tendência de escoamento do ar foi nas colunas (direção x) formadas entre os paletes, na direção adjacente ao fluxo dos sopradores, pois nas linhas (direção y) formadas entre estes, o ar apresentou as menores velocidades e em certas regiões tendeu a estagnação (figuras 7 e 8). O caso 2 apresentou diferenças na distribuição do fluxo de ar em relação ao caso 1 onde as linhas de paletes foram retiradas, o baixo fluxo nestas regiões foi o fator aparente que diferenciou os dois casos, pois a variação de temperaturas em certas áreas foi maior que $2^{\circ} \mathrm{C}$ (figura 8). Estes resultados apresentaram concordância com o trabalho realizado por Damasceno et al. (2013) que chegaram a conclusão que os campos de velocidade e temperatura se relacionam diretamente e as regiões com menor fluxo de ar podem desfavorecer a troca de calor convectiva do meio e como consequência causar focos de maior temperatura, dilatando o tempo necessário para se atingir a temperatura final desejada e também os gastos energéticos do processo. 


\section{CONCLUSÃO}

A simulação via fluidodinâmica computacional acusou as variações da temperatura em certas regiões, relacionadas às variações de velocidade de escoamento do ar e da quantidade de carga presente na câmara. O uso de CFD pode colaborar para a previsão de gastos energéticos e até possíveis mudanças na carga ou equipamento para otimização do processo.

\section{REFERENCIAS}

ASHRAE. Handbook of Refrigeration. Atlanta: American Society of Heating, Refrigerating, and Air-Conditioning Engineers, 2010

CHOURASIA, M. K.; GOSWAMI, T. K. Steady state CFD modeling of airflow, heat transfer and moisture loss in a commercial potato cold store. International Journal of Refrigeration, v. 30, n. 4, p. 672-689, 2007.

DAMASCENO, G. P; CORRÊA, J. L. G; NASCIMENTO, F. R. Determinação via cfd de campos de velocidade e temperatura em uma câmara de armazenamento. In: CONGRESSO BRASILEIRO DE SISTEMAS PARTICULADOS: ENEMP, XXXVI., 2013, Maceió. Anais... Maceió: Edufal, 2013 (in Press).

DELELE, M. A.; SCHENK, A.; TIJSKENS, E.; RAMON, H.; NICOLAÏ, B. M.; VERBOVEN, P. Optimization of the humidification of cold stores by pressurized water atomizers based on a multiscale CFD model. Journal of Food Engineering, v. 91, n. 2, p. 228-239, 2009.

DELELE, M. A.; NGCOBO, M. E. K.; OPARA, U. L.; MEYER, C. J. Investigating the Effects of Table Grape Package Components and Stacking on Airflow, Heat and Mass Transfer Using 3-D CFD Modelling. Food and Bioprocess Technology, v. 6, n. 9, p. 2571-2585, 2013.

HO, S. H.; ROSARIO, L.; RAHMAN, M. M. Numerical simulation of temperature and velocity in a refrigerated warehouse. International Journal of Refrigeration, v. 33, n. 5, p. 1015-1025, 2010.

MENTER, F. R. Zonal two equation k- $\omega$ turbulence models for aerodynamic flows. AIAA 24th Fluid Dynamics Conference, v., n., p., 1993.

SOFTWARE ANSYS CFX. Version 11.0. ANSYS Europe Ltd., 2007. 1 DVD-ROM. Help menu index.

XIE, J.; QU, X.-H.; SHI, J.-Y.; SUN, D.-W. Effects of design parameters on flow and temperature fields of a cold store by CFD simulation. Journal of Food Engineering, v. 77, n. 2, p. 355363, 2006.

\section{AGRADECIMENTOS}

Agradecemos ao apoio financeiro da FAPEMIG, CAPES e CNPq. 